Fig. 2-An example of a computer display produced by one of the latest types of easy-to-use software that generates graphical control displays and data viewing windows in a multi-computer, networked control system for medium-tolarge physics experiments. It shows some of the objects that are employed to build up control windows graphically.

The window at the bottom left is the tool-box window of the graphical editor. This is used to

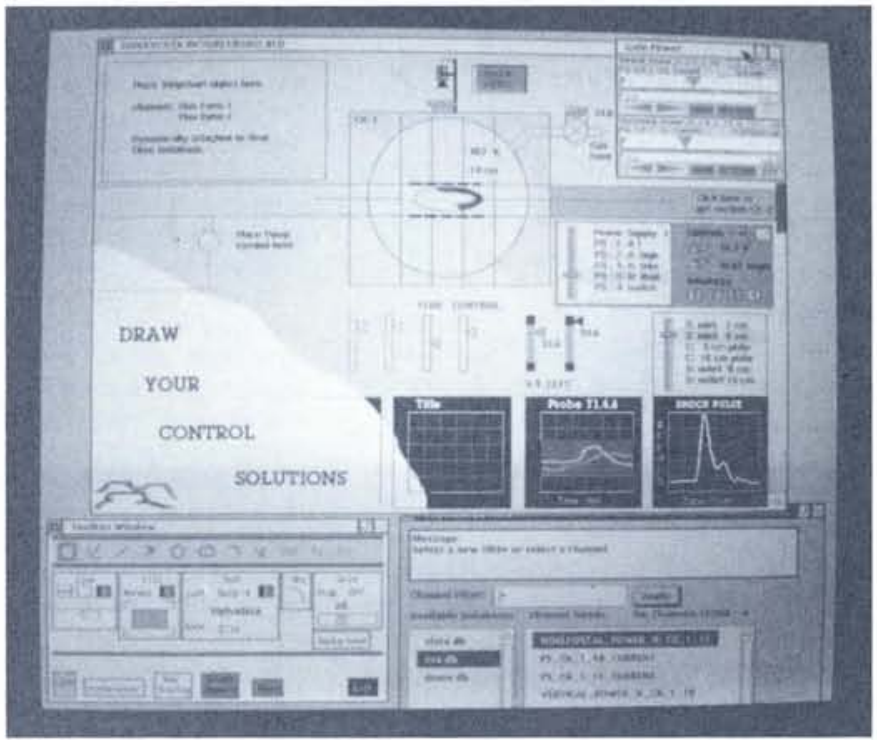

create the background drawing and to define and place the objects in the window that will be animated by connection to the real-time database. Other objects and active areas can be defined to control the windows and the overall operation of the control system.

At the upper right is a small slider bank which allows for the display and control of analogue values. Selection switches, bars and graphs can also be seen. Many other tools are available for use from the tool-box. In the bottom right is a separate window for viewing and changing the real-time databases, which include e.g. the channel descriptions. creation of graphical control displays and data viewing windows in a multi-computer, networked system (Fig. 2).

\section{Conclusions}

Computing is developing to provide the user with a more homogeneous working environment. This is also true for accelerator control systems, which are destined in the 1990's to become more integrated with physics design and simulation. This will allow greater understanding and automation of accelerators, resulting in advances in accelerator science and technology with reduced cost and risk.

\section{REFERENCES}

[1] Norbeck E., Proc. Conf. on the Utilization of Multiparameter Analyzers in Nuclear Physics, Grosinger, NY, 1962, p. 56.

[2] Puttnam T.M., Jameson R.A. and Schultheis T.M., IEEE Trans. NS-12 (1965) 21.

[3] Daneels A. and Skarek P., Nucl. Inst. Methods A247 (1986) 141.

[4] Clout P. N. et al., Nucl. Inst. Methods A247 (1986) 116.

[5] Clout P.N., Nucl. Inst. and Methods A293 (1990) 456 .

\title{
Pulse-To-Pulse Modulation
}

\section{R. Steiner \\ GSI, Darmstadt, Germany}

Associate Member of EPS

Accelerator performance for bunched particle beams has been enhanced significantly by modifying the beam properties on a pulse-to-pulse basis.

For continuous operation, a pulse is defined as the complete acceleration cycle in which the the properties of a bunch of charged particles of the same kind are changed. The pulse-to-pulse modulation (PPM) technique allows the uninterrupted production of beams of different particles with different characteristics in a single machine. It was, to the best of my knowledge, applied [1] for the first time in CERN's Proton Synchrotron complex (see overleaf).

Pulse-to-pulse-modulation is a powerful means to optimize the efficiency of pulsed accelerators. Hence:

- PPM provides beams to more than one user at virtually the same time.

- Many accelerators serve as injectors for another machine which is, as a rule, both larger and slower than the injector by about one order of magnitude. An injector,

Rudolf Steiner has a Diploma in physics from the University of Mainz, Germany. $\mathrm{He}$ is the Project Leader of the SIS/ESR Control Systems Group at GSI, Darmstadt where he was formerly the head of the electronics laboratory. for instance, is typically occupied with injection into the next machine for some $10 \%$ of the time: PPM releases the remaining $90 \%$ of the injector's beam time for other experiments with beams that may even be different to the injection beam. - Accelerators used in medicine exploit PPM for optimal performance by ensuring equal dose deposition in tissue.

Ideal PPM operation means that an arbitrary number of different acceleration cycles may be executed in an arbitrary sequence. For practical reasons, the number of different cycles is limited to a reasonable number, say 8 or 16 . The same is true for the "arbitrary" sequence: a limited number of cycles, say 256, forms a "supercycle" which is executed periodically. In spite of these restrictions, PPM has a considerable impact on the design of the control and timing systems of an accelerator facility.

\section{Impact on Equipment Control}

For PPM operation, the equipment control level (the lowest level in the control system) must be intelligent to provide flexibility, interrupt driven to provide speed and a precise response time, and equipped with enough memory to store the complete datasets of operating conditions (magnet ramps, etc.) of different cycles.
Security mechanisms must make sure that datasets are complete and consistent after operator interaction and before execution. Equipment controllers must be prepared to execute any legal function of a supercycle at any legal time. They must respond to coded interrupts, called events, as the number of legal functions will exceed the number of interrupt levels of the processors. An event may be a code (e.g. a 16-bit number) together with an interrupt request signal: it must contain information about the action to be performed (e.g. "start cycle"), the type of cycle, and eventually the supercycle.

Providing the complex, time dependent variations in the operating state needed for PPM on the equipment control level in pure hardware would be troublesome so intelligence must inevitably be placed at the local level.

\section{Impact on the Timing System}

A timing system is needed to generate events and to distribute them to equipment controllers. A bus system (see page 35), instead of the provision of individual signals for hundreds of separate pieces of equipment, is the most convenient approach for distributing events as the latter is inflexible and requires too much hardware. The events must occur in an orderly sequence: the timing system must only start actions that can be executed without conflicts. Once again, this can hardly be achieved without local intelligence.

\section{Conflicts}

However, the more flexible a timing system, the more sensitive it is to conflict 
PPIM At CERN

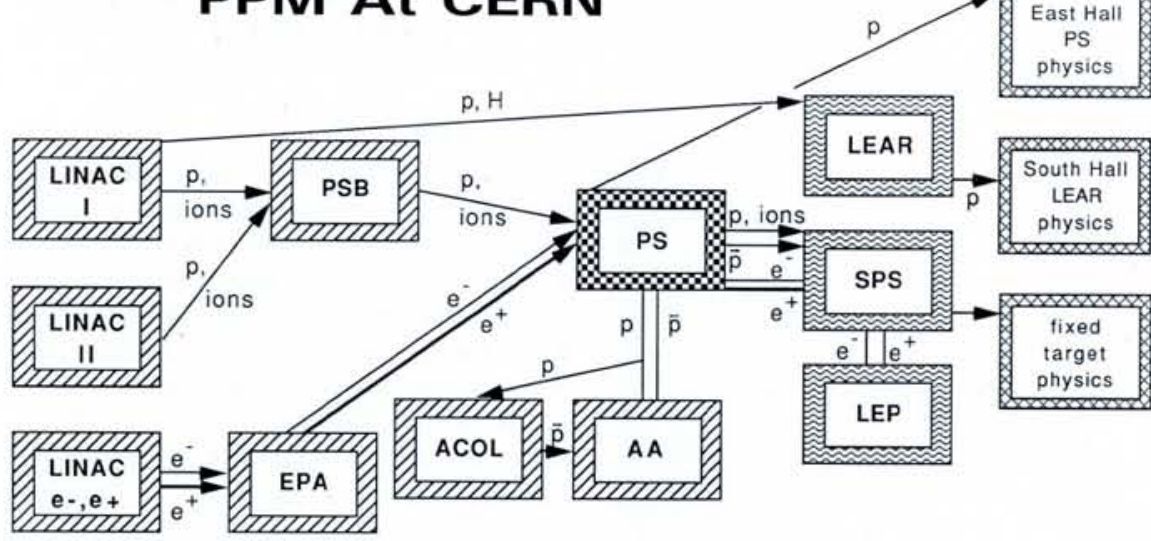

The application of pulse-to-pulse modulation (PPM) to CERN's PS accelerator complex. Upper: the input and output particle beams originating from and passing through the various accelerators (boxes) before they are delivered to physics experiments. The PS must accelerate beams with different characteristics involving different injection and outgoing lines in well-defined time intervals reserved for each beam.

Lower left: A snapshot of a PS supercycle divided into a number of cycles, each of which repeatedly delivers particle of an indicated energy and intensity (particles/ $\mathrm{cm}^{3}$ ) to a user specified in the same column. The profiles at the top represent the field in the synchrotron's main magnets.

Lower right: On each console the operator has to first select the "virtual accelerator" he wants to interact with. Any of his actions will then only be applied to those cycles of a supercycle which comply with the selection, e.g. to the fourth in the figure for the third console from the left.
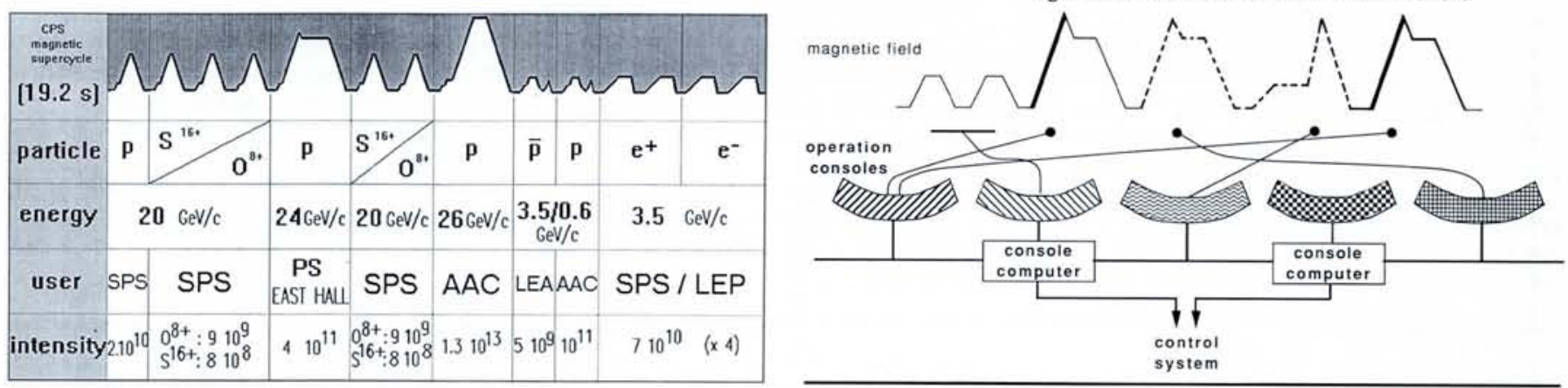

conditions. The two main sources of conflicts (they cannot be eliminated with software checks) are asynchronous inputs from the accelerator to the timing system; and asynchronous request signals to synchronize different accelerators (e.g. for injection) when the accelerators are running on different timing signals. A possible approach to eliminate conflicts uses acceptance windows where timing signals are only valid during specified intervals.

\section{Synchronization}

Synchronizing different machines may be more tricky. Imagine a particle storage ring calling for injection about every hour. The injector will execute it's supercycle hundreds of times without an injection pulse. When injection is requested, the supercycle must be modified as quickly as possible without operator intervention. A property called execution tag (ET) is assigned to any execution pattern of a cycle in the supercycle. An injection cycle can be programmed for any legal time window with, for instance, an ET value defining the no operation value, by setting its ET to the ET value of the appropriate injection cycle. The ET value is reset to the original value after beam transfer, and the injector continues operating without wasting time.

\section{Impact on the Operating Level}

A major task in a PPM environment is the programming of the timing system. Individual cycles (i.e. sequences of events) must be constructed and then put together to form a supercycle. Nothing runs
Fig. 1 - An example of a typical display at an operator's console of the SIS control system during pulse-topulse modulation of the operation conditions. Selection of the type of cycle within a supercycle for adjustment or examination is by choosing a number from 1 to 15 in the horizontal row. Values in the aktiv, setting and reading $\mathrm{co}$ lumns refer to slave properties of devices (properties that change from cycle to cycle): the columns power, reset and init are for master properties that remain unchanged.

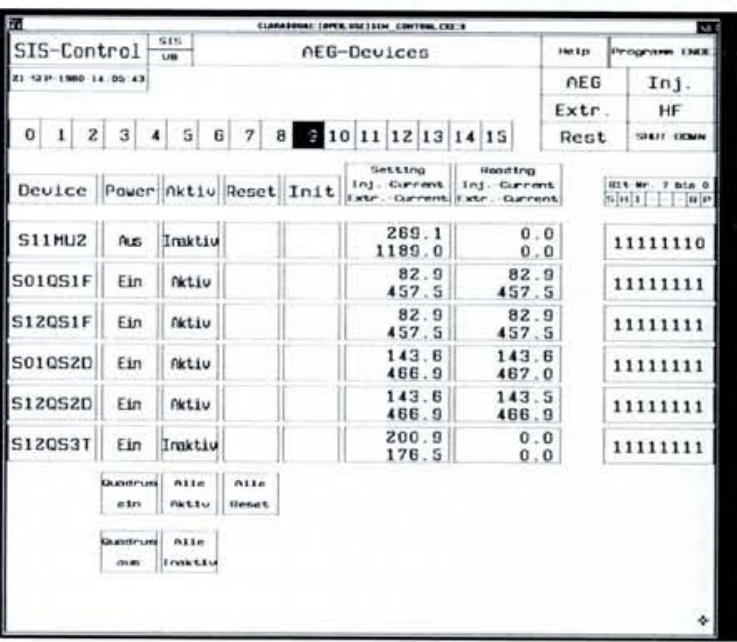

properly if the event sequences are inconsistent so, although specialists are responsible, programming the supercycle should be supported by operating programs: privileged access to the operating programs, and consistency checks on e.g. setting values are also essential.

Having set the timing systems, most of the operating actions in a PPM system deal with a specific type of cycle in the supercycle. Classifying device properties according to master properties that do not depend on special cycles, e.g. on/off, and slave properties that refer to one special cycle, e.g. settings, is very convenient. For instance, a useful slave property is one called "'active/inactive" where all actions of particular equipment items are suppressed in one cycle but left active in another. This distinction shows up on the operator's display, e.g. Fig. 1 for the SIS accele- rator/storage ring, where selecting a number from the line " $0-15$ " specifies the type of cycle within the supercycle to be examined or acted upon. Values in the "setting" and "reading" column and the choice in the "aktiv" column only refer to slave properties for the selected cycle: "power", "reset" and "init" define master properties valid for all types of cycles. Note, however, that status bits mix master and slave properties: ramping the field of a magnet may be correct in one type of cycle but represent a fault in another.

\section{Conclusions}

Pulse-to-pulse modulation of a particle accelerator clearly affects almost every controls component, from the equipment interface to the operator's interface. PPM should thus be carefully considered during the design phase of a control system. 
It helps to pay considerable attention to: strategies for solving timing conflicts; starting PPM operation with a comfortable operating software for the timing system to guarantee consistent event sequences; implementing diagnostics for fault conditions (e.g. "event overrun", "event sequence error", "event collision") as early as possible even if you are sure that these things will never happen in your system; considering the use of event filters for the equipment controllers (i.e. pass only meaningful events to the processors since the number of events in a cycle tends to increase steadily so the controllers may become overloaded with the handling of useless requests).

PPM should be implemented from the very beginning as it is tricky if not impossible to upgrade a single-cycle machine to a PPM machine without a complete redesign of the controls and timing system. Nonetheless, upgrades for PPM are underway (e.g. AGS, Brookhaven, USA [2]).

And never forget that while it is fun to operate an accelerator with PPM, it is no fun at all with a poorly designed timing system, poor software support and poor diagnostic tools.

\section{REFERENCES}

[1] Boillot J. et al., IEEE Trans. Nucl. Sci. NS-28 (1981) 2195.

[2] Barton D.S., Nucl. Inst. and Methods A293 (1990) 210

\section{L’ÉCOLE POLYTECHNIQUE FÉDÉRALE DE LAUSANNE}

met au concours un poste de

\section{PROFESSEUR DE PHYSIQUE EXPÉRIMENTALE}

Le nouveau professeur sera rattaché à I'Institut de physique expérimentale du Département de physique, qui a une activité principale de recherche en physique de la matière condensée. II développera une recherche fondamentale et appliquée dans le domaine des

\section{PHÉNOMÈNES DE SURFACE ET/OU MATÉRIAUX GRANULAIRES}

Les domaines de recherche principaux de I'Institut de physique expérimentale sont l'étude des propriétés physiques d'agrégats en jets ou supportés et des propriétés structurales et dynamiques de surfaces et couches minces. Les candidats qui ont un intérêt en physique fondamentale et appliquée dans les domaines de la physique des surfaces / dynamique de l'interaction gazsurface / matériaux nanophases / "cluster assembled materials" sont invités à faire acte de candidature.

Le nouveau professeur donnera des enseignements de base en physique à des étudiants de diverses sections d'ingénieurs de I'EPFL, ainsi que des enseignements dans son domaine de spécialité.

Délai d'inscription: 31 mai 1991.

Entrée en fonction: Printemps 1992 ou à convenir.

Les personnes intéressées voudront bien demander les formules de candidature au:

Secrétariat général de I'Ecole Polytechnique Fédérale de Lausanne, CE Ecublens, CH - 1015 LAUSANNE/Suisse

\section{Expand Your Data Acquisition Capabilities...}

...With Our Powerful New MicroVAX On A CAMAC Module!

Our Model 3968 Intelligent CAMAC Crate Controller is a front-end processor featuring...

- A 2.7 VUP, $20 \mathrm{MHz}$ rtVAX 300 computer!

- 4 Mbytes on-board memory!

- Floating point coprocessor!

- Ethernet port with networking software!

- A console terminal port \& optional SCSI port!

- VAXELN target software license!

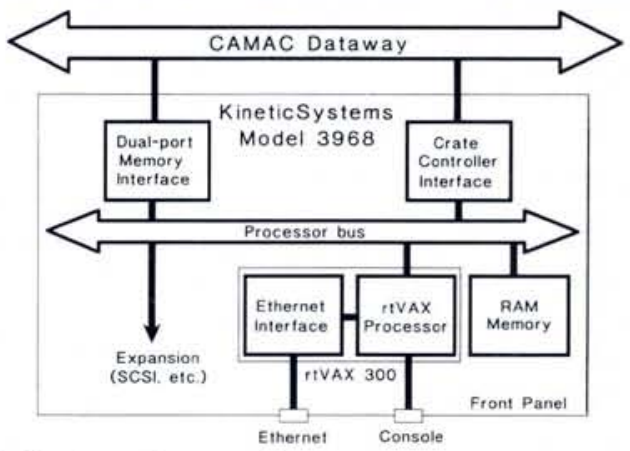

\section{...And With Our Versatile New VME Interface!}

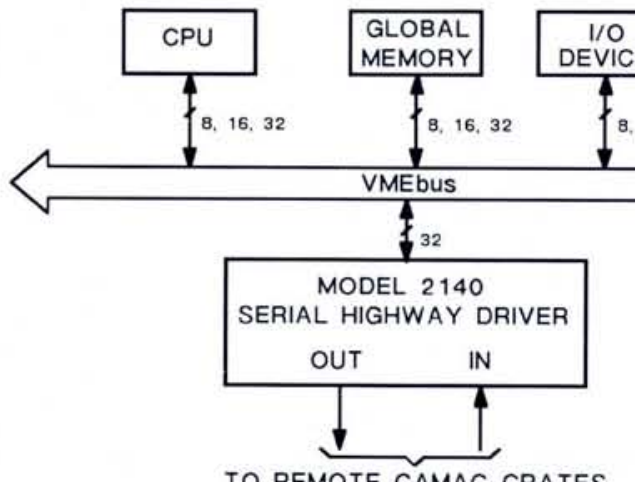

Kinetic Systems International S.A.

TO REMOTE CAMAC CRATES 